\title{
MiRNA seed shifting: origin and functional implications
}

\author{
P. Vorozheykin ${ }^{1 *}$, N. Yazikov ${ }^{1}$, I. Titov $^{1,2}$ \\ ${ }^{1}$ Novosibirsk State University, Novosibirsk, Russia \\ ${ }^{2}$ Institute of Cytology and Genetics SB RAS, Novosibirsk, Russia \\ *e-mail: pavel.vorozheykin@gmail.com
}

Key words: miRNA, seed shifting, targets, miRNA origin, isomiRs, miRNA biogenesis

Motivation and Aim: miRNAs constitute an extensive class of small RNAs. Mostly the miRNAs suppress the undesirable transcripts and genetic materials. Nucleotides 2-8 of the miRNA (so-called "seed" region) play a crucial role in the target recognition and binding; due to the imperfect binding rules one miRNA can regulate expression of many genes and thus control many biological functions. With the growth of deep-sequencing data, a diversity of miRNA location, length and sequence have been discovered (socalled isomiRs).

Results: To make out the isomiR role in the miRNA life cycle we consider the causes of the miRNA variants (evolutionary changes, processing mechanisms [1] and annotation errors [2]) and their functional implications. During the evolution the accumulated mutations in the pri-/pre-/miRNA change the miRNA boundaries (including "seed" location), sequence and secondary structure of the precursor. Other reasons of miRNA end heterogeneity are an inaccuracy of processing by the RNase proteins or by the splicing, their predisposition to the precursor sequence and structure and possible interconnection between Dicer cleavage errors and the length of the pre-miRNA hanging ends $[1,3]$. The considered changes can lead to the new biological functions of miRNA and diverse levels of miRNA expression.

Conclusion: The results obtained in the work can be useful to predict new miRNAs and their functions based on the similarity to the existing ones. They allow to take a fresh look at the evolution of the silencing and can help to reconsider the role of the miRNA seed and the rest of the miRNA sequence and its precursor.

\section{References}

1. Titov I.I., Vorozheykin P.S. (2018). Comparing miRNA structure of mirtrons and non-mirtrons. BMC Genomics. 19(3):114.

2. Fromm B., Billipp T., Peck L.E. et al. (2015). A uniform system for the annotation of vertebrate microRNA genes and the evolution of the human microRNAome. Annu. Rev. Genet. 49:213-242.

3. Starega-Roslan J., Witkos T.M., Galka-Marciniak P., Krzyzosiak W.J. (2015). Sequence features of Drosha and Dicer cleavage sites affect the complexity of isomiRs. Int. J. Mol. Sci. 16(4):8110-8127. 